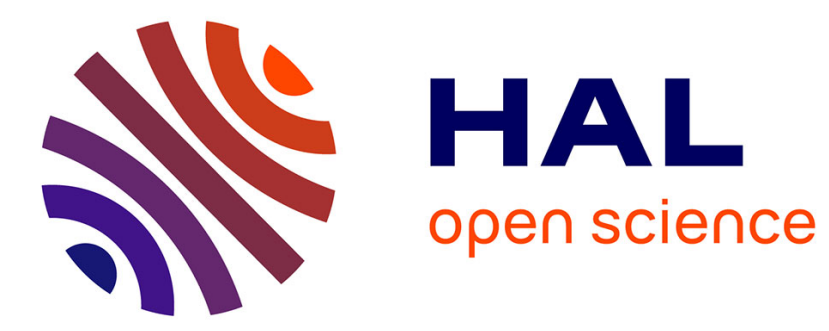

\title{
Realistic Prediction of BER and AMC with MRC Diversity for Indoor Wireless Transmissions
}

Meiling Luo, Guillaume Villemaud, Jialai Weng, Jean-Marie Gorce, Jie Zhang

\section{To cite this version:}

Meiling Luo, Guillaume Villemaud, Jialai Weng, Jean-Marie Gorce, Jie Zhang. Realistic Prediction of BER and AMC with MRC Diversity for Indoor Wireless Transmissions. Wireless Communications and Networking Conference (WCNC), 2013 IEEE, Apr 2013, Shanghai, China. 10.1109/WCNC.2013.6555227 . hal-00920929

\section{HAL Id: hal-00920929 \\ https://hal.inria.fr/hal-00920929}

Submitted on 19 Dec 2013

HAL is a multi-disciplinary open access archive for the deposit and dissemination of scientific research documents, whether they are published or not. The documents may come from teaching and research institutions in France or abroad, or from public or private research centers.
L'archive ouverte pluridisciplinaire $\mathbf{H A L}$, est destinée au dépôt et à la diffusion de documents scientifiques de niveau recherche, publiés ou non, émanant des établissements d'enseignement et de recherche français ou étrangers, des laboratoires publics ou privés. 


\title{
Realistic Prediction of BER and AMC with MRC Diversity for Indoor Wireless Transmissions
}

\author{
Meiling Luo*†, Guillaume Villemaud ${ }^{\dagger}$, Jialai Weng ${ }^{\ddagger}$, \\ Jean-Marie Gorce ${ }^{\dagger}$, Jie Zhang* ${ }^{* \ddagger}$ \\ *Ranplan Wireless Network Design Ltd. \\ Suite 25, The Business Competitiveness \\ Centre, Kimpton Road, LU2 0SX \\ Luton, United Kingdom \\ †Université de Lyon \\ INRIA \\ INSA-Lyon, CITI \\ F-69621, Villeurbanne, FRANCE \\ $\ddagger$ Department of Electronic and \\ Electrical Engineering \\ University of Sheffield, Mappin Street \\ Sheffield, S1 3JD, United Kingdom
}

\begin{abstract}
Bit Error Rate (BER) is a key parameter used to evaluate the performance of radio communication systems. In this paper, a realistic BER of radio MRC diversity systems is predicted based on the Multi-Resolution Frequency Domain ParFlow (MRFDPF) model. The prediction takes into account the correlations among diversity branches and makes no assumptions of the correlation model. The predicted realistic BER for MRC diversity systems can be very useful to many wireless applications, such as, adaptive modulation and coding (AMC) scheme, or optimal power allocation.
\end{abstract}

\section{INTRODUCTION}

Radio channels are subject to reflection, refraction and diffraction, etc caused by the surrounding obstacles or objects. Hence, multipath propagation exists and these multipath radio waves can interfere with each other constructively or destructively, resulting in fast and random fluctuation of the received signal strength. The fast and random fluctuation of the received signal strength is usually called fading. Diversity technique [1] is introduced to mitigate the effects of fading by making use of several copies of the signal, which experience independent or approximately independent fading, to reduce the probability of simultaneous deep fades. Space diversity is one of the diversity techniques which uses multiple antennas to improve the quality of the radio channels. In space diversity systems, several combining schemes are used to recover the desired signal, such as, Maximum Ratio Combining (MRC), Equal Gain Combining (EGC) and Selection Combining (SC) [1][2]. Among the three, MRC yields the highest Signal-toNoise Ratio (SNR), thus it is the optimum combiner, but also, it is the most complicated one [2].

When analyzing the performance of diversity systems, the assumption usually made is that the received signals at diversity branches are independent and identically distributed (i.i.d) [2]. However, in real-life situations, a completely i.i.d fading can rarely be achieved due to e.g. the nature of propagation environments and/or insufficient antenna separation. For instance, when there is a dominant Line-of-Sight (LOS) signal in the propagation, the received signal will be Rice distributed and then the received signals at diversity branches will not be independent, but correlated with each other. In this situation, the maximum theoretical diversity gain can not be achieved and it is necessary to consider the impact of correlation. The impact of correlation on the performance of diversity systems has received extensive attention over the past several decades [2][3][4][5][6][7][8]. Some correlation models have been proposed in [4][5] and they have been adopted by many authors to analyze the performance of diversity systems [5][6][7][8].

In this paper, we predict the Bit Error Rate (BER) with considering the correlations among diversity branches for indoor wireless environments based on the deterministic channel model, the Multi-Resolution Frequency Domain ParFlow (MRFDPF) model. The predicted BER is accurate and realistic because no assumptions have been made of the correlation model. We compute the correlation coefficients among diversity branches based on the MR-FDPF simulation, i.e. we do not use the existing assumptive correlation models.

The rest of the paper is structured as follows. First, section II gives a brief introduction of the MR-FDPF model, followed by the BER analysis with MRC diversity with considering the correlations among branches in section III. Then section IV presents the estimation of the Nakagami-m fading parameter. Finally, section V shows the results, and conclusion is drawn in section VI.

\section{THE MR-FDPF MODEL}

The MR-FDPF model is a deterministic radio propagation model. It simulates radio wave propagation according to the nature of radio waves. For example, when radio electromagnetic waves meet a surface, they will be reflected, and when they encounter an obstacle, diffractions will occur. Deterministic channel models include all these propagation phenomena naturally, thus they are usually very accurate, which is not the case for empirical models.

The MR-FDPF model is proposed by Gorce et al. in [9] and it is proven to be accurate and efficient in predicting not only the mean power [9][10], but also a number of fading statistics [11][12][13][14]. The MR-FDPF model is the ParFlow model [15] in the frequency domain which is a sort of finite difference models. The ParFlow model assumes that the electric field in the discrete space can be divided into four directive flows as shown in Fig. 1. The flows which take energy into the space 


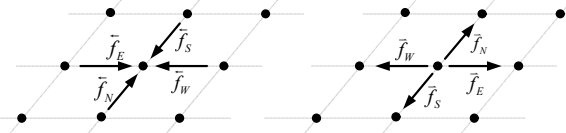

Fig. 1. The inward flows and outward flows associated with each space node

node are called the inward flows and the flows which take energy out are called the outward flows. The inward flows and outward flows are updated alternately in the discrete time based on the local scattering equation depending on the properties of materials, e.g. concrete, wood or air. The ParFlow model needs a sufficient time iterations to reach the steady state, but when we do it in the frequency domain, like the MR-FDPF model, the steady state problem is directly solved. Furthermore, for radio wave propagation in indoor environments, the MRFDPF model is advantageous over Ray-optical models [16] since it includes all the effects of materials in a natural way, which means its computational effort is independent of the complexity of propagation environments.

\section{BER ANALYSIS WITH MRC DIVERSITY}

BER acts as an indication of the quality of radio links and it is a key parameter for higher layer applications, e.g. adaptive modulation and coding (AMC) scheme or optimal power allocation. For instance, AMC dynamically adapts its modulation and coding scheme based on the BER. Therefore, the accuracy of the prediction of BER is very crucial.

Theoretically, the average BER over fading channels is obtained by averaging the corresponding BER of Additive White Gaussian Noise (AWGN) channels over the Probability Density Function (PDF) of the instantaneous SNR which depends on the fading [2]. That is

$$
P_{b: \text { fading }}(E)=\int_{0}^{\infty} P_{b: A W G N}(E ; \gamma) P_{\gamma}(\gamma) d \gamma
$$

where $\gamma \triangleq \alpha^{2} E_{b} / N_{0}$ denotes the instantaneous SNR per bit, $\alpha$ is the fading amplitude, $E_{b}$ is the signal energy per bit, $N_{0}$ is the noise power spectral density, $P_{\gamma}(\gamma)$ is the PDF of the instantaneous SNR depending on the fading, $P_{b: \text { fading }}(E)$ is the BER over fading channels and $P_{b: A W G N}(E ; \gamma)$ is the BER over AWGN channels conditioned on the fading. $P_{b: A W G N}(E ; \gamma)$ is obtained by replacing $E_{b} / N_{0}$ by $\gamma$ in the BER over AWGN channels $P_{b: A W G N}(E)$.

Normally, the $P_{b: A W G N}(E)$ is a Gaussian Q-function for ideal coherent detection. On the other hand, the Moment Generating Function (MGF) $M_{\gamma}(s)$ associated with a specific fading is defined by [2]

$$
M_{\gamma}(s)=\int_{0}^{\infty} e^{s \gamma} \cdot P_{\gamma}(\gamma) d \gamma
$$

Using the definition of the MGF together with the desired form of the Gaussian Q-function instead of the classic form, which is

$$
Q(x)=\frac{1}{\pi} \int_{0}^{\pi / 2} \exp \left(-\frac{x^{2}}{2 \sin ^{2} \theta}\right) d \theta
$$

we come to a unified and simplest way for computing the $P_{b: \text { fading }}(E)$ since the MGFs of a number of fading models are already available. Then typically, the $P_{b \text { :fading }}(E)$ is just a single integral with finite limits and integrand containing the MGF of the fading.

One point we should emphasize is that the $P_{b: A W G N}(E)$ here which is used to compute the $P_{b \text { :fading }}(E)$ should not be the commonly used approximate AWGN BER obtained from the approximate relationship between the BER $P_{b}(E)$ and Symbol Error Rate (SER) $P_{s}(E)$ as follows

$$
P_{b}(E) \cong \frac{P_{s}(E)}{\log _{2} M}
$$

because this approximation is only valid for large symbol SNR, but the limits of the integral in equation (1) are from zero to infinity (i.e. $\gamma$ can vary from zero to infinity over fading channels). The $P_{b: A W G N}(E)$ used here should be either the exact AWGN BER or the approximate AWGN BER which is accurate at both low and high SNR.

Here, we adopt the approximate AWGN BER of Lu et al. [17] as the $P_{b: A W G N}(E)$ to compute the $P_{b \text { :fading }}(E)$ since it is very accurate at both low and high SNR and it is much simpler than the exact AWGN BER [18][19][20][21]. Thus, for M-PSK modulations, we have

$$
\begin{aligned}
P_{b: A W G N}(E) & \cong \frac{2}{\max \left(\log _{2} M, 2\right)} . \\
& \sum_{i=1}^{\max \left(\frac{M}{4}, 1\right)} Q\left(\sqrt{\frac{2 E_{b} \log _{2} M}{N_{0}}} \sin \frac{(2 i-1) \pi}{M}\right)
\end{aligned}
$$

where $M$ is the modulation order, e.g. $M=2$ for BPSK, $M=4$ for QPSK. For M-QAM modulations, we have

$$
\begin{aligned}
& P_{b: A W G N}(E) \cong \frac{4}{\log _{2} M}\left(1-\frac{1}{\sqrt{M}}\right) \cdot \\
& \sum_{i=1}^{\sqrt{M} / 2} Q\left((2 i-1) \sqrt{\frac{3 E_{b} \log _{2} M}{N_{0}(M-1)}}\right)
\end{aligned}
$$

Therefore, for the BER for the M-PSK modulations over fading channels, we have

$$
\begin{aligned}
& P_{b: \text { fading }}(E) \cong \frac{2}{\max \left(\log _{2} M, 2\right)} \cdot \\
& \sum_{i=1}^{\max \left(\frac{M}{4}, 1\right)} \frac{1}{\pi} \int_{0}^{\pi / 2} M_{\gamma}\left(-\frac{\log _{2} M}{\sin ^{2} \theta} \sin ^{2} \frac{(2 i-1) \pi}{M}\right) d \theta
\end{aligned}
$$

For the BER for the M-QAM modulations over fading channels, we have

$$
\begin{aligned}
P_{b: \text { fading }}(E) & \cong \frac{4}{\log _{2} M}\left(1-\frac{1}{\sqrt{M}}\right) \cdot \\
& \sum_{i=1}^{\sqrt{M} / 2} \frac{1}{\pi} \int_{0}^{\pi / 2} M_{\gamma}\left(-\frac{(2 i-1)^{2} \cdot 3 \cdot \log _{2} M}{2 \sin ^{2} \theta \cdot(M-1)}\right) d \theta
\end{aligned}
$$

For the case where MRC diversity is used, the computation of $P_{b: \text { fading }}(E)$ depends on the MGF of the combined SNR 


$$
M_{\gamma}(s)=\prod_{d=1}^{D}\left(1-\frac{s \bar{\gamma}_{d}}{m}\right)^{-m} \cdot\left\lfloor\left.\left[\begin{array}{cccc}
1 & \sqrt{\rho_{12}}\left(1-\frac{m}{s \bar{\gamma}_{2}}\right)^{-1} & \cdots & \sqrt{\rho_{1 D}}\left(1-\frac{m}{s \bar{\gamma}_{D}}\right)^{-1} \\
\sqrt{\rho_{12}}\left(1-\frac{m}{s \bar{\gamma}_{1}}\right)^{-1} & 1 & \cdots & \sqrt{\rho_{2 D}}\left(1-\frac{m}{s \bar{\gamma}_{D}}\right)^{-1} \\
\cdot & \cdot & \cdot \\
\cdot & \cdot & \cdot \\
\cdot & \cdot & \cdot \\
\sqrt{\rho_{1 D}}\left(1-\frac{m}{s \bar{\gamma}_{1}}\right)^{-1} & \sqrt{\rho_{2 D}}\left(1-\frac{m}{s \bar{\gamma}_{2}}\right)^{-1} & \cdots & \cdot
\end{array}\right]\right|_{D \times D} ^{-m}\right.
$$

PDF. Since we want the BER to have a high level of accuracy, we try to make as less assumptions as possible. Thus, here we consider the diversity reception with $D$ nonidentically distributed branches with arbitrary correlation. More specifically, we assume that the diversity branches share the same Nakagami-m fading parameter $m$, but they can each have their own average SNR $\bar{\gamma}_{d}(d=1,2, \cdots, D)$. The envelope correlation coefficient between any channel pairs $\left(d, d^{\prime}\right)$ $\left(d, d^{\prime}=1,2, \cdots, D\right)$ is denoted by $\rho_{d d^{\prime}}$. Then, the MGF of the combined SNR PDF over the Nakagami-m fading channels is given by equation (9) (at the top of this page) [3], where $|[M]|_{D \times D}$ stands for the determinant of the $D \times D$ matrix $M$. Substituting the equation (9) into the equation (7) and equation (8), we can easily obtain the $P_{b \text { :fading }}(E)$ for the M-PSK and M-QAM modulations.

\section{ESTIMATION OF THE NAKAGAMI-M FADING PARAMETER}

\section{A. Estimation methods for the $m$ parameter}

The Nakagami-m fading distribution is given as follows [22]

$$
P_{\alpha}(\alpha)=\frac{2 m^{m} \alpha^{2 m-1}}{\Omega^{m} \Gamma(m)} \exp \left(-\frac{m \alpha^{2}}{\Omega}\right), \quad \alpha \geq 0
$$

where $\Omega=\mathrm{E}\left(\alpha^{2}\right)$ is the average power of the fading and $m$ is called the Nakagami-m fading parameter. The Nakagami$\mathrm{m}$ distribution has been received extensive attention since it gives the best fit to many measurement data, such as, landmobile and indoor-mobile multipath propagation [2][23]. The parameter $m$ varies from $\frac{1}{2}$ to $\infty$ indicating the severity of the fading. The smaller the $m$ value, the severer the fading. The Nakagami-m distribution includes Rayleigh distribution when $m=1$ and one-sided Gaussian distribution when $m=\frac{1}{2}$ as special cases. When $m \rightarrow \infty$, the Nakagami-m fading channel approaches to a nonfading AWGN channel. When $m>1$, we have a one-to-one mapping between Nakagami-m distribution and Rice distribution by

$$
\mathrm{m}=\frac{(1+K)^{2}}{1+2 K}, \quad K \geq 0
$$

where $K$ is the Rice $\mathrm{K}$ factor. Hence, the Nakagami-m fading model can describe a very wide range of multipath fading phenomena.

There exist a number of methods for estimating the Nakagami-m fading parameter $m$ [24][25][26][27][28]. From the equation (10), we know that the $m$ parameter can be estimated by estimating the Rice $\mathrm{K}$ factor first, and then map it to the $m$ parameter. On the other hand, we can also estimate the $m$ parameter by the Greenwood's method [27] since it outperforms other estimators [25]. The Greenwood's method is stated briefly as follows [25]

$$
\hat{m}=\left\{\begin{array}{l}
\frac{0.5000876+0.1648852 y-0.0544274 y^{2}}{y}, \\
0<y \leq 0.5772 \\
\frac{8.898919+9.059950 y+0.9775373 y^{2}}{y\left(17.79728+11.968477 y+y^{2}\right)} \\
0.5772<y<17
\end{array}\right.
$$

where $y=\ln \left(\hat{\mu}_{2} / G\right), \ln (\cdot)$ denotes the natural logarithm operator, $\hat{\mu}_{2}$ is the arithmetic mean of sample power, i.e. $\hat{\mu}_{2}=\frac{1}{N} \sum_{n=1}^{N} x_{n}^{2}$, and $G$ is the geometric mean of sample power $G=\left(\prod_{n=1}^{N} x_{n}^{2}\right)^{1 / N}$

\section{B. Remarks of goodness of fit tests}

The goodness of fit tests measure how well the distribution you selected fits to your sample data. The KolmogorovSmirnov test [29] is one of the widely used goodness of fit tests. It tries to seek the biggest difference between the empirical distribution function (EDF) $F_{n}(x)$ and the hypothetical distribution function $F(x)$. The Kolmogorov-Smirnov test has the advantage that there is no need to make any assumptions of the distribution of the sample data, but in return, it has the disadvantage that the hypothetical distribution function $F(x)$ should be fully specified, otherwise, e.g. if any parameters need to be estimated from the sample data, the result of the test will be conservative [30]. For more details, please refer to [14].

In this paper, we want to test the Nakagami-m distribution with the unknown $m$ parameter. To the best of our knowledge, this type of goodness of fit test problem is not available from any existing goodness of fit tests, so we still adopt the Kolmogorov-Smirnov test to conduct the goodness of fit test, but just notice that the results are conservative.

\section{Results And Discussion}

\section{A. The scenario}

The indoor simulation scenario used in the MR-FDPF model is the CITI building at INSA-Lyon, France. The simulation is performed at $3.5 \mathrm{GHz}$ with the space discretization step of $2 \mathrm{~cm}$. The transmitter is an omnidirectional antenna which is placed in an office of the CITI building marked by Tx both in 


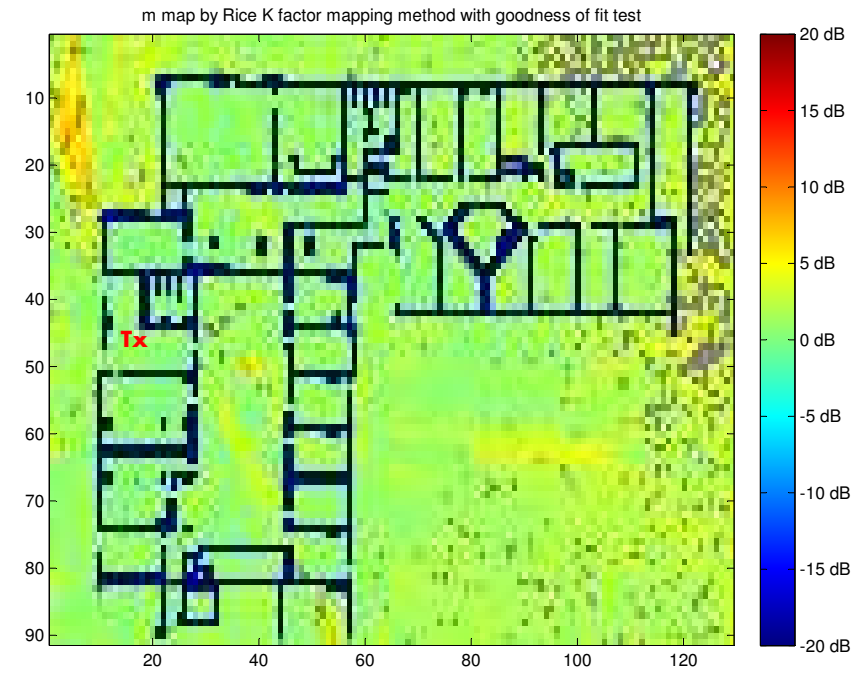

Fig. 2. The $m$ map by the Rice $\mathrm{K}$ factor mapping method with goodness of fit test.

Fig. 2 and Fig. 3. The Equivalent Isotropically Radiated Power (EIRP) of the transmitter is $23 \mathrm{dBm}$.

\section{B. $m$ parameter map of Nakagami-m fading channels}

As stated in section IV, although there exist a number of estimation methods for the $m$ parameter of the Nakagami-m fading, we adopt here two methods: One is the Rice $\mathrm{K}$ factor mapping method since we have already estimated the Rice $\mathrm{K}$ factor in [14]. The other one is the Greenwood's method since it outperforms others. The $m$ parameter maps extracted from the MR-FDPF model by above-mentioned two methods are given in $\mathrm{dB}$ in Fig. 2 and Fig. 3, respectively. In the two figures, each $m$ parameter is obtained over a local area with dimensions $23 \times 23$ pixels, but only half of them taken based on an alternating pattern are chosen to implement the estimations since only uncorrelated samples are needed. The space discretization step of the MR-FDPF simulation is $2 \mathrm{~cm}$, and the uncorrelated distance is 0.38 wavelength according to [31], which is about $3.26 \mathrm{~cm}$ at $3.5 \mathrm{GHz}$, thus each pixel in the simulation is uncorrelated with its second neighbors.

\section{Performance evaluation of the estimation of the $m$ param- eter of the Nakagami-m fading}

We conduct the goodness of fit test to evaluate the estimation performance of the Nakagami-m fading parameter by the two above-mentioned methods. The Kolmogorov-Smirnov test is adopted and performed at the $5 \%$ significance level. The grey points in Fig. 2 and Fig. 3 denote the locations where the estimated $m$ parameter has failed the Kolmogorov-Smirnov test. From the Fig. 2, we see that most of the estimated $m$ values by the Rice $\mathrm{K}$ factor mapping method have passed the Kolmogorov-Smirnov test, which validates that the RICEFIT method developed by Ridgway [32] works well in estimating the Rice $\mathrm{K}$ factor. Especially, the locations of the failed $m$ values are basically in accordance with those of the failed Rice $\mathrm{K}$ values where the envelope distributions there are supposed to

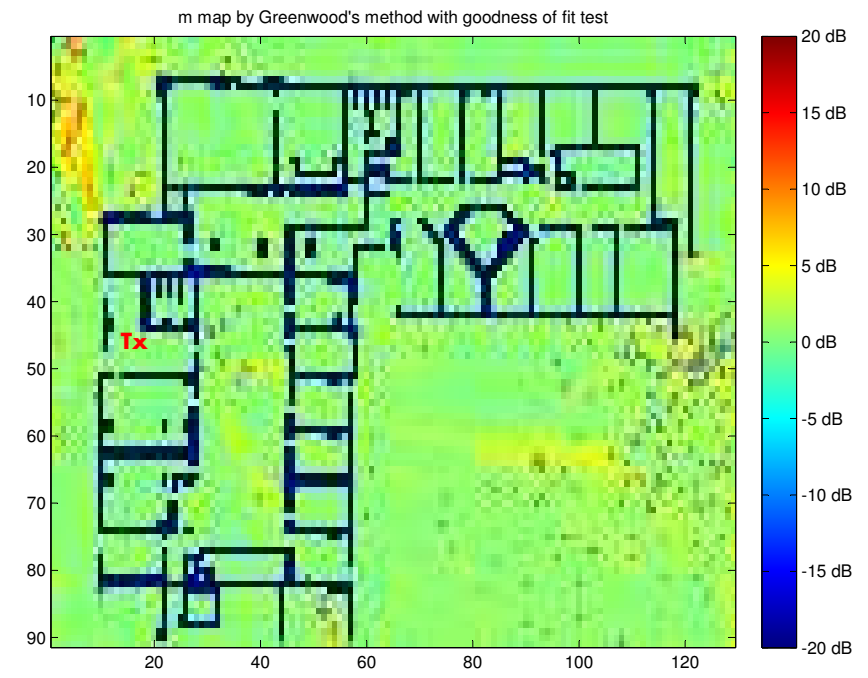

Fig. 3. The $m$ map by the Greenwood's method with goodness of fit test.

not retain very strong Rice characteristics [14]. At the end, it is observed in Fig. 3 that the locations of the failed $m$ values are very few, which further proves that the Greenwood's method is among the best available methods for estimating the $m$ parameter of the Nakagami-m fading. Therefore, in this paper, we use the Greenwood's method to estimate the $m$ parameter for the following BER prediction.

The mean value of the estimated $m$ parameter by the Greenwood's method is 1.2854 , and the variance is 0.4778 . The mean value of 1.2854 indicates that the propagation environment under investigation is a Rayleigh-like environment.

\section{Prediction of $B E R$ and $A M C$ with $M R C$ diversity}

The BER performance of the real-life diversity systems depends not only on the SNR, but also on the fading parameters and the correlations among diversity branches. Since it is possible to estimate the fading parameters and compute the correlations among diversity branches based on the MR-FDPF model, the predicted BER from the MR-FDPF model should be realistic and accurate. The correlations among branches are computed based on the space samples of the signal amplitude around the corresponding antennas from the MR-FDPF model. We compute the BER for the BPSK, QPSK, 16QAM and 64QAM modulation schemes according to the equation (7) and equation (8). Due to the limited space, we just take the QPSK modulation for example, we show the BER maps for QPSK modulation with the $1 \times 3$ diversity, $1 \times 2$ diversity and SISO system in Fig. 4, Fig. 5 and Fig. 6, respectively. In these three figures, the BER is computed based on the thermal noise of $3.5 \mathrm{MHz}$ bandwidth. It is observed that the BER difference between the $1 \times 2$ diversity and SISO system is larger than that between the $1 \times 3$ diversity and 1x2 diversity. This is reasonable because for diversity systems, the BER decreases exponentially with the number of diversity branches.

For the AMC, we consider only the target uncoded BER of $10^{-3}$ to adapt the modulation schemes. Fig. 7, Fig. 8 and 


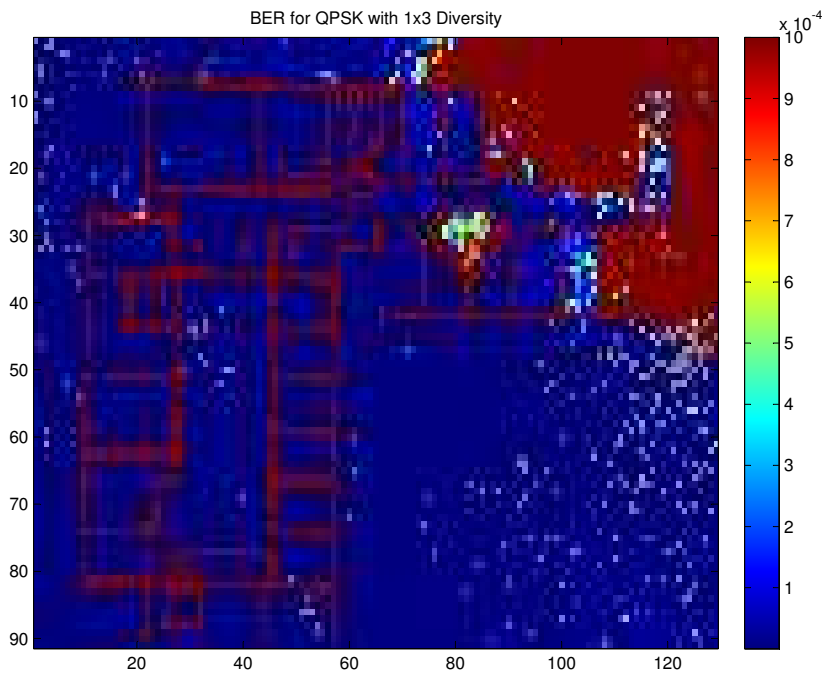

Fig. 4. The BER map for QPSK with 1x3 Diversity.

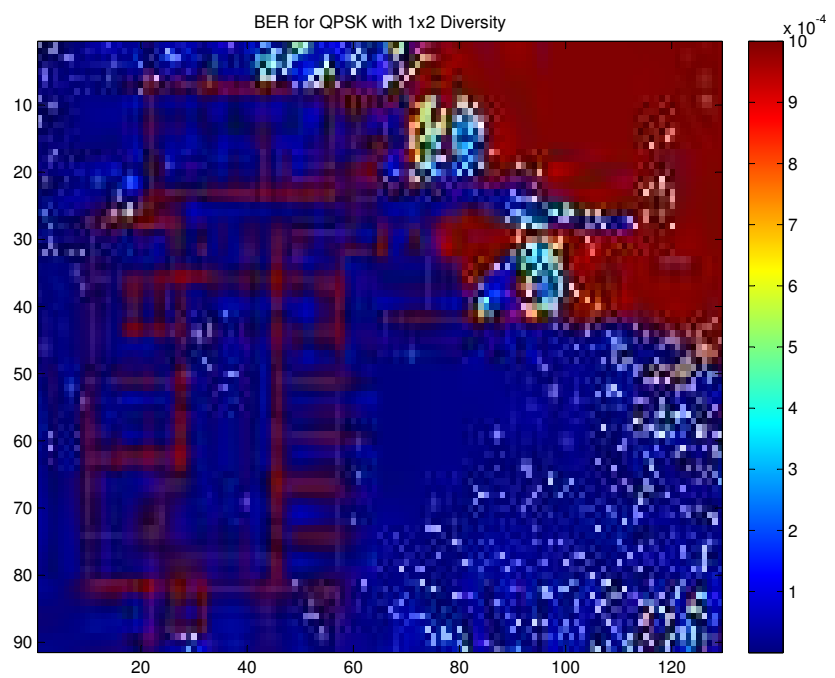

Fig. 5. The BER map for QPSK with 1x2 Diversity.

Fig. 9 show the modulation maps with the $1 \times 3$ diversity, $1 \times 2$ diversity and SISO system, respectively. The numbers in the three figures: 1, 2, 3 and 4 denote the BPSK, QPSK, 16QAM and 64QAM, respectively. The number 0 denotes none of these modulation schemes above can achieve the target BER. From the Fig. 4 - Fig. 9, we can see how much the diversity can improve the system performance.

\section{E. Discussion}

A realistic prediction of BER with MRC diversity can be achieved by considering the fading parameters and correlations among diversity branches. Note that we have assumed here a frequency non-selective fading over the whole bandwidth of 3.5 MHz. For a frequency selective fading channel, a fine analysis of the channel frequency characteristics can be conducted with the wideband extension of the MR-FDPF model [33]. Then sub-carrier AMC can be applied to wideband OFDM

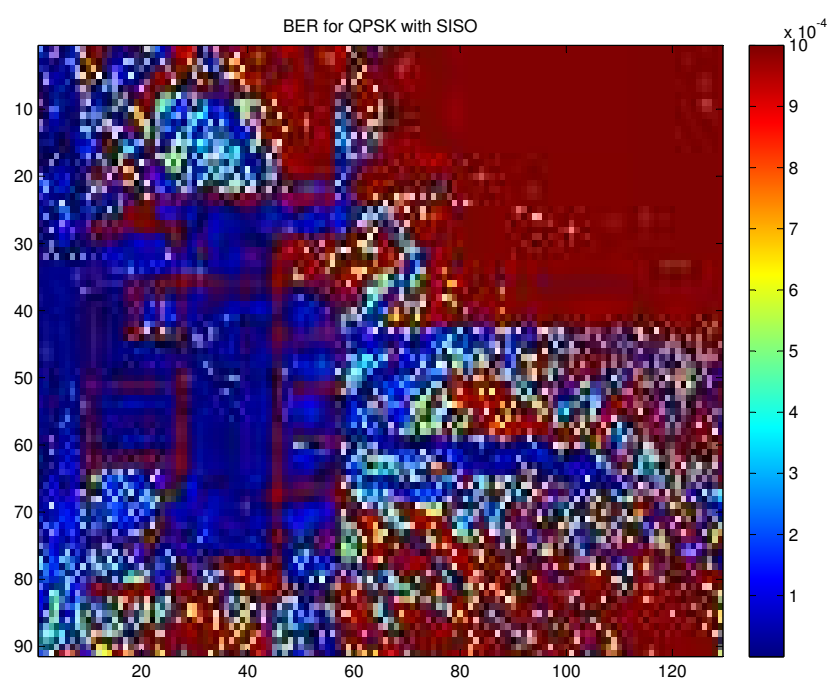

Fig. 6. The BER map for QPSK with SISO systems.

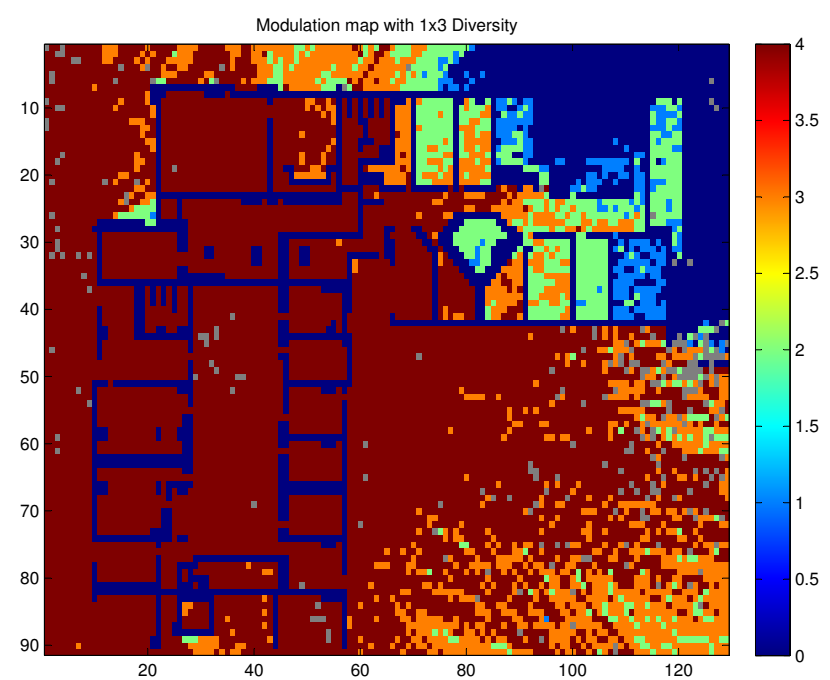

Fig. 7. The modulation map with $1 \times 3$ diversity with BER threshold $10^{-3}$

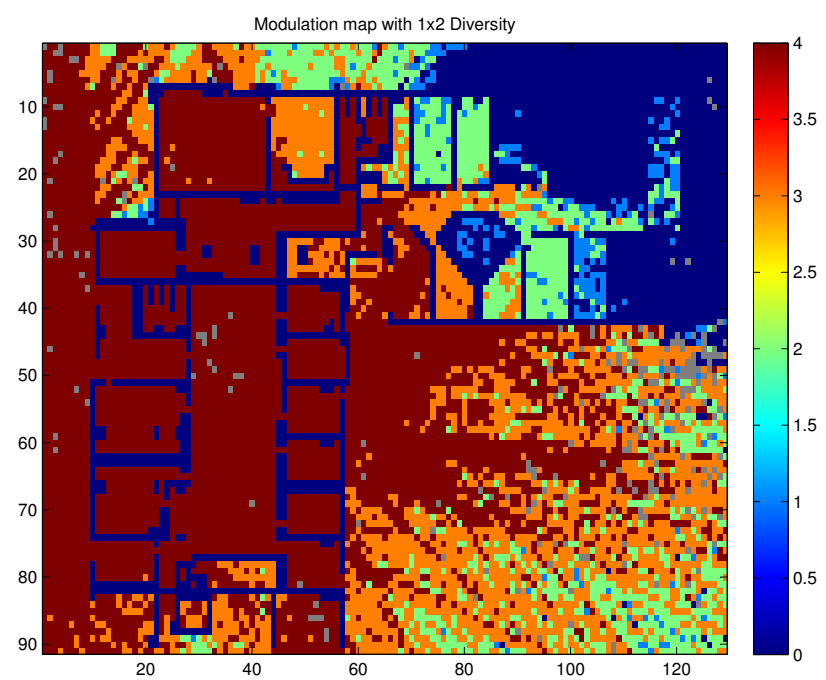

Fig. 8. The modulation map with $1 \times 2$ diversity with BER threshold $10^{-3}$ 


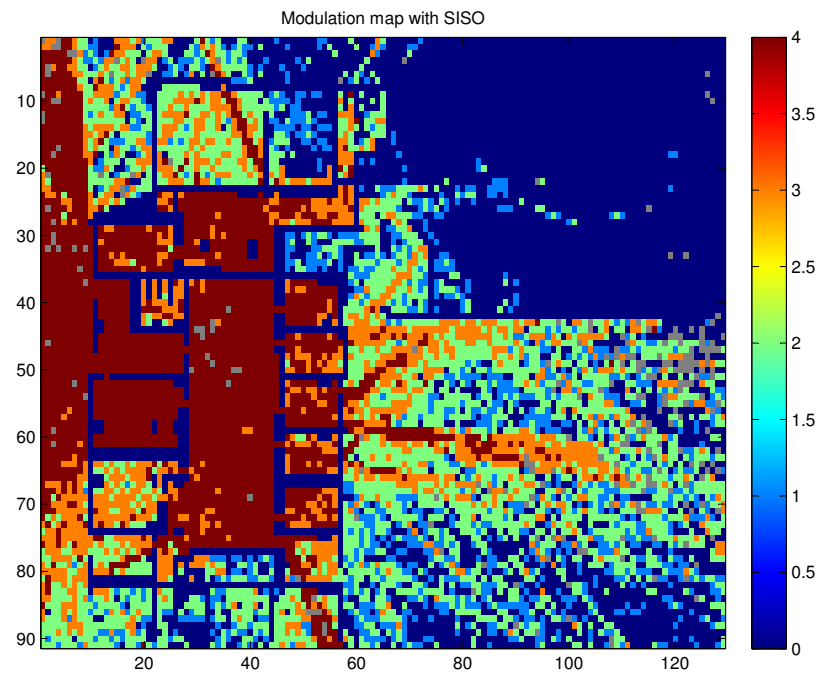

Fig. 9. The modulation map with SISO with BER threshold $10^{-3}$.

systems and finally the aggregate throughputs of multi-rate transmissions of wideband OFDM systems can be predicted.

\section{CONCLUSION}

A realistic BER and AMC with MRC diversity for indoor wireless transmission is predicted based on the deterministic MR-FDPF model. The prediction takes into account not only the SNR, but also the fading severity and the correlations among diversity branches. The estimated fading parameter has been verified by the goodness of fit test. The predicted realistic BER can be very useful to AMC and other wireless applications.

\section{ACKNOWLEDGMENT}

This work is funded by the FP7 IPLAN Project.

\section{REFERENCES}

[1] D. Tse and P. Viswanath, Fundamentals of wireless communication. Cambridge university press, 2005.

[2] M. Simon and M. Alouini, Digital communication over fading channels Wiley-IEEE Press, 2004, vol. 86

[3] M. Alouini and M. Simon, "Multichannel reception of digital signals over correlated nakagami fading channels," in Proceedings of the Annual Allerton Conference on Communication Control and Computing, vol. 36. Citeseer, 1998, pp. 146-155.

[4] J. Pierce and S. Stein, "Multiple diversity with nonindependent fading," Proceedings of the IRE, vol. 48, no. 1, pp. 89-104, 1960.

[5] V. Aalo, "Performance of maximal-ratio diversity systems in a correlated nakagami-fading environment," IEEE Transactions on Communications, vol. 43, no. 8, pp. 2360-2369, 1995.

[6] Q. Zhang, "Maximal-ratio combining over nakagami fading channels with an arbitrary branch covariance matrix," IEEE Transactions on Vehicular Technology, vol. 48, no. 4, pp. 1141-1150, 1999.

[7] P. Lombardo, G. Fedele, and M. Rao, "MRC performance for binary signals in nakagami fading with general branch correlation," IEEE Transactions on Communications, vol. 47, no. 1, pp. 44-52, 1999.

[8] F. Patenaude, J. Lodge, and J. Chouinard, "Noncoherent diversity reception over nakagami-fading channels," IEEE Transactions on Communications, vol. 46, no. 8, pp. 985-991, 1998.

[9] J.-M. Gorce, K. Jaffres-Runser, and G. de la Roche, "Deterministic approach for fast simulations of indoor radio wave propagation," IEEE Transactions on Antennas and Propagation, vol. 55, no. 3, pp. 938 -948, 2007.
[10] K. Runser and J.-M. Gorce, "Assessment of a new indoor propagation prediction method based on a multi-resolution algorithm," in IEEE 61st Vehicular Technology Conference, VTC 2005-Spring, vol. 1, 2005, pp. $35-38$ Vol. 1.

[11] M. Luo, D. Umansky, G. Villemaud, M. Lafort, and J.-M. Gorce, "Estimating channel fading statistics based on radio wave propagation predicted with deterministic MR-FDPF method," in 5th European Conference on Antennas and Propagation (EuCAP 2011), Rome, Italy, April, 2011.

[12] M. Luo, G. De La Roche, G. Villemaud, J. Gorce, D. Umansky, and J. Zhang, "Simulation of wide band multipath fast fading based on finite difference method," in IEEE Vehicular Technology Conference, no. 2011, 2011.

[13] M. Luo, N. Lebedev, G. Villemaud, G. De La Roche, J. Zhang, and J. Gorce, "On predicting large scale fading characteristics with the MR-FDPF method," in 6th European Conference on Antennas and Propagation (EUCAP). IEEE, 2012, pp. 2186-2190.

[14] M. Luo, G. Villemaud, J. Gorce, and J. Zhang, "Realistic prediction of BER and AMC for indoor wireless transmissions," IEEE Antennas and Wireless Propagation Letters, vol. 11, pp. 1084 - 1087, 2012.

[15] P. Luthi, "Lattice wave automata: from radio wave to fracture propagation," Ph.D. dissertation, Computer Science Department, University of Geneva, 24 rue General-Dufour, 1211 Geneva 4, Switzerland, 1998.

[16] C. Balanis, Advanced Engineering Electromagnetics. New York, NY, USA: John Wiley \& Sons, 1999.

[17] J. Lu, K. Letaief, J. Chuang, and M. Liou, "M-PSK and M-QAM BER computation using signal-space concepts," IEEE Transactions on Communications, vol. 47, no. 2, pp. 181-184, 1999.

[18] P. Lee, "Computation of the bit error rate of coherent m-ary psk with gray code bit mapping," IEEE Transactions on Communications, vol. 34, no. 5, pp. 488-491, 1986.

[19] J. Lassing, E. Strom, E. Agrell, and T. Ottosson, "Computation of the exact bit-error rate of coherent $\mathrm{m}$-ary psk with gray code bit mapping," IEEE Transactions on Communications, vol. 51, no. 11, pp. 1758-1760, 2003.

[20] D. Yoon, K. Cho, and J. Lee, "Bit error probability of m-ary quadrature amplitude modulation," in IEEE 52nd Vehicular Technology Conference, VTC-Fall, 2000, vol. 5. IEEE, 2000, pp. 2422-2427.

[21] K. Cho and D. Yoon, "On the general BER expression of one-and twodimensional amplitude modulations," IEEE Transactions on Communications, vol. 50, no. 7, pp. 1074-1080, 2002.

[22] M. Nakagami, "The m-distribution-A general formula of intensity distribution of rapid fading," Statistical Method of Radio Propagation, 1960.

[23] A. Sheikh, M. Abdi, and M. Handforth, "Indoor mobile radio channel at $946 \mathrm{mhz}$ : Measurements and modeling," in 43rd IEEE Vehicular Technology Conference, 1993. IEEE, 1993, pp. 73-76.

[24] A. Abdi and M. Kaveh, "Performance comparison of three different estimators for the nakagami $\mathrm{m}$ parameter using monte carlo simulation," IEEE Communications Letters, vol. 4, no. 4, pp. 119-121, 2000.

[25] Q. Zhang, "A note on the estimation of nakagami-m fading parameter," Communications Letters, IEEE, vol. 6, no. 6, pp. 237-238, 2002

[26] H. Thom, "A note on the gamma distribution," Monthly Weather Review, vol. 86, no. 4, pp. 117-122, 1958.

[27] J. Greenwood and D. Durand, "Aids for fitting the gamma distribution by maximum likelihood," Technometrics, vol. 2, no. 1, pp. 55-65, 1960.

[28] K. Bownan and L. Shenton, "Properties of estimators for the gamma distribution," History and Philosophy of Logic, vol. 11, no. 4, pp. 377519, 1982.

[29] R. D'Agostino and M. Stephens, Goodness-of-fit techniques. New York: Marcel Dekker, 1986, vol. 68

[30] F. Massey Jr, "The kolmogorov-smirnov test for goodness of fit," Journal of the American Statistical Association, pp. 68-78, 1951.

[31] J. Parsons, The mobile radio propagation channel. Wiley Online Library, 2000, vol. 81.

[32] G. Ridgway, "RICEFIT," [Online] http://www.mathworks.com/matlab central/fileexchange/14237-ricerician-distribution/content/rician/ricefit.m.

[33] J.-M. Gorce, G. Villemaud, and P. Flipo, "On simulating propagation for OFDM/MIMO systems with the MR-FDPF model," in the Fourth European Conference on Antennas and Propagation (EuCAP),, 2010, pp. $1-5$. 